\title{
Assorted Facets of Physical Layer in Cognitive Radio: A Review
}

\author{
Seema Verma \\ Associate Professor, \\ Dept. of Electronics, \\ Banasthali Vidyapeeth, \\ Rajasthan, India
}

\author{
Ghanshyam Choudhary \\ Assistant Professor, \\ Dept. of Electronics,MNIT, \\ Jaipur, Rajasthan, India
}

\author{
Pallavi Yarde \\ Assistant Professor, \\ Dept. of Electronics, \\ Manipal University, \\ Jaipur, Rajasthan, India
}

\begin{abstract}
The intensification in the number of mobile subscribers over the preceding years leads to a situation of the Voice-oriented, data-oriented wireless telephony. It is now point in time to explore new demands and to find the new ways to broaden the mobile concepts. The Cognitive Radio (CR) is one of the essential steps that initiate the solution towards these problems. Then also there are lot of challenges associated to Cognitive Radio in the area of operational field and implementation in Real world. In this paper we tried to give the ideas about the voyage of Cognitive Radio from evolution to challenges experienced with respect to Physical Layer.
\end{abstract}

\section{General Terms}

Wireless Communication, Physical Layer, Cognitive Radio, Spectrum Sensing.

\section{Keywords}

Cognitive Radio, Cognitive Radio Architecture, Cognitive Process/Cycle, Modulation Schemes, Spectrum sensing.

\section{INTRODUCTION}

The radio spectrum popularly known as Electromagnetic Spectrum is a natural resource for communication. The use of the resource is licensed by the government agencies. Presently the utilization of this allotted spectrum is a big challenge as the numbers of mobile users are growing continuously. As the number of application analogous to wireless communication increased that causes the lack of spectrum availability called Spectrum Scarcity. The situation with allocated bands is like some bands are unutilized, some are partially utilized and some are fully utilized at an instant. So it is major duty to find out the unused and partially used bands and allocate those bands to the application which seeks for it, without interfering to the other applications that are already in use. Cognitive radio (CR) is seen as one of the most encouraging approaches to solving the Spectrum Scarcity problem by allowing smart and dynamic spectrum management in future wireless communication systems. CR is characterized as a system, able to adapt its transmission or reception functions on the basis of cognitive interaction with the wireless environment in which it operates. It is observed that utilization of application in licensed band is lower as compared to unlicensed band applications [11]. To understands the different phases of Cognitive Radio one has to go through the various issues related to it. Every system has its own architecture and organization and both of these are interrelated to each other. The architecture defines what components it constitutes and organization defines the interrelation between the available components and their behavior in different situations.

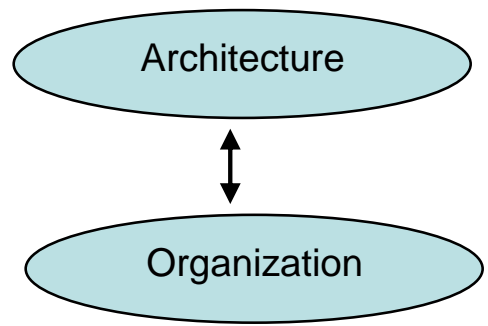

Fig. 1: Relation between architecture and organization

\section{COGNITIVE RADIO TYPES}

Depending on the set of parameters taken in to account in deciding on transmission and reception changes, the Cognitive Radio is categorized as:

\subsection{Full Cognitive Radio}

Every possible parameter is noticeable by a wireless entity or network is considered.

\subsection{Spectrum Sensing Cognitive Radio \\ Only the radio frequency spectrum is considered.}

The Cognitive Radio can also be categorized according to spectrum available:

\subsection{Licensed Band Cognitive Radio}

Cognitive Radio is capable of using bands assigned to licensed users (Paid users); apart from unlicensed bands.

\subsection{Unlicensed Band Cognitive Radio}

It can only utilize the unlicensed band of radio spectrum.

\section{COGNITIVE RADIO ARCHITETURE}

Every Cognitive Radio system has its own layered architecture. Each layer of the system has specific roles. Ashwin Amanna and Jeffrey H. Reed gives the Survey of Cognitive Radio Architecture[2], that CR Architecture based on the classification of CR framework and literatures like Mitola's Cognitive Radio Architecture[3], Public Safety Cognitive Radio technology has been applied to the interoperability needs of public safety [4]. The architectural view can be seen in the fig.2, shows that the Cognitive Radio Architecture classified according to different frameworks and literature work. 


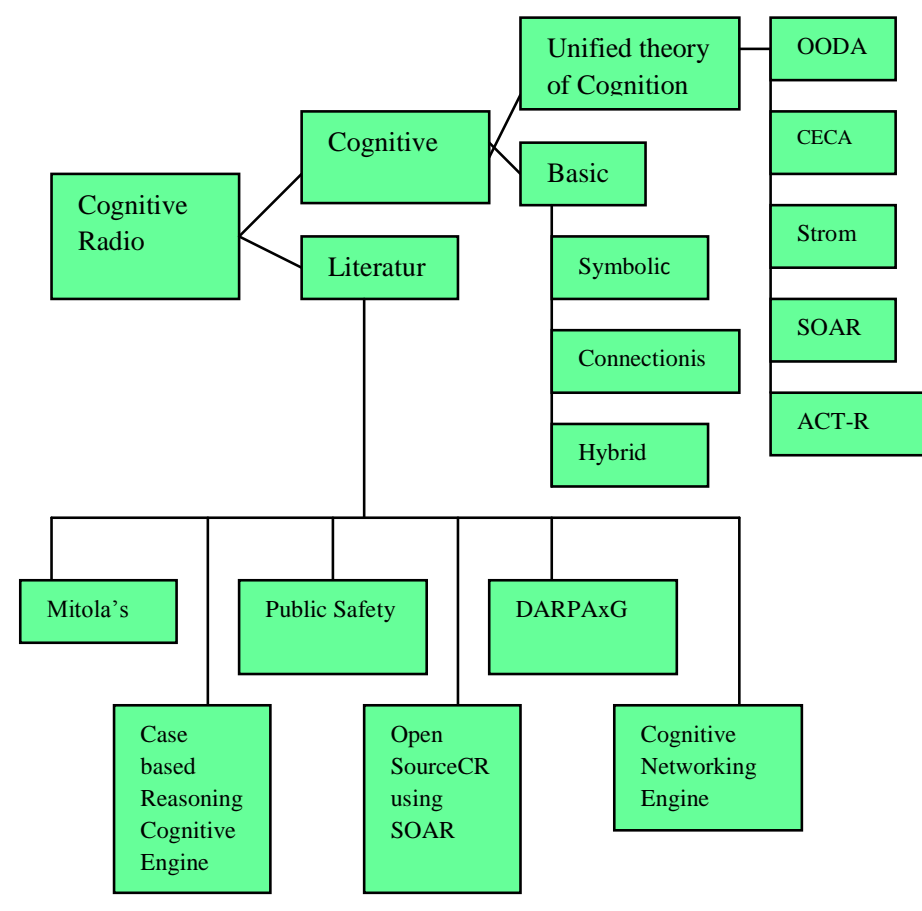

Fig.2: Cognitive Radio Architecture Classification

Fig. 3 gives the Cognitive Radio layered architecture gives by Yang Xiao, Fei Hu [1]. It shows CR works on 3 layers. The very first or bottom layer is PHY (Physical) layer, responsible for the Spectrum available, Spectrum Sensing, Modeling \& Forecasting of Spectrum and Coordination between the spectrum utilization. These all functionalities of PHY layer are come under the category called Spectrum Sensing. This category is used to identify the underutilized sub-bands.

The second layer is MAC (Media Access Control) layer, responsible for allocation of the spectrum that is available at the time of requirement. This is done with the help of the protocols available at MAC layer like Partially Observable Markov Decision Process (POMDP), for the synchronization Cognitive-MAC (CMAC) protocol, Dynamic Open Spectrum Sharing MAC Protocol (DOSS)[5] etc. Another responsibility of MAC layer is Adaptive MAC design that involves the Spectrum Mapping, Negotiations and Data Transfer. The term Adaptive MAC design specifies the designing approach based on the priori data information obtained from reliable source.

The third and final layer is Routing layer. It is basically defined for Ad-hoc networks to route the data from one node to another one. These routing strategies [5],[6],[7] are based on different parameters like traffic load in the network, Reliability of the working node and the network, Battery Autonomy and last but not the least end-to-end delay. These are considered as Cognitive Routing Cost Functions (CRCFs).

\section{COGNITIVE PROCESS}

As a seismic shift in the wireless communications and networking world, CR could potentially break the ever-serious "bottleneck" of limited spectrum availability and open up new frontiers of opportunities for wireless system designers and application developers. The whole task of Cognitive Radio is divided into number of sub-tasks and collectively called as "Cognitive Process" or "Cognitive Cycle". Amna Saad Kamil, Ibrahim Khider, gives Cognitive Radio Process Fig.4 [8] that explains cognitive Radio process has 3 basic steps for communication.

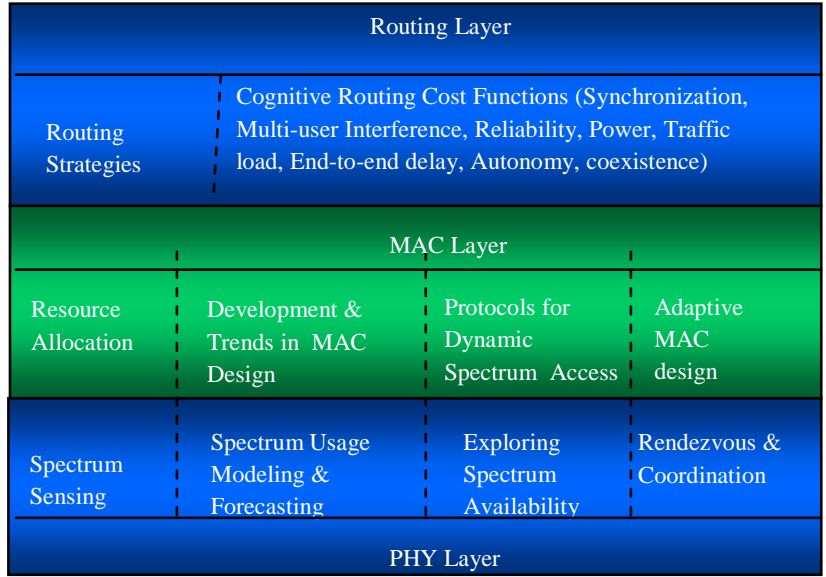

Fig.3: Layered Architecture of Cognitive Radio

- $\quad$ Spectrum Sensing

- Spectrum Analysis

- Spectrum Decision

The main aim of using these steps is to find out the Spectrum Holes commonly known as White Spaces.

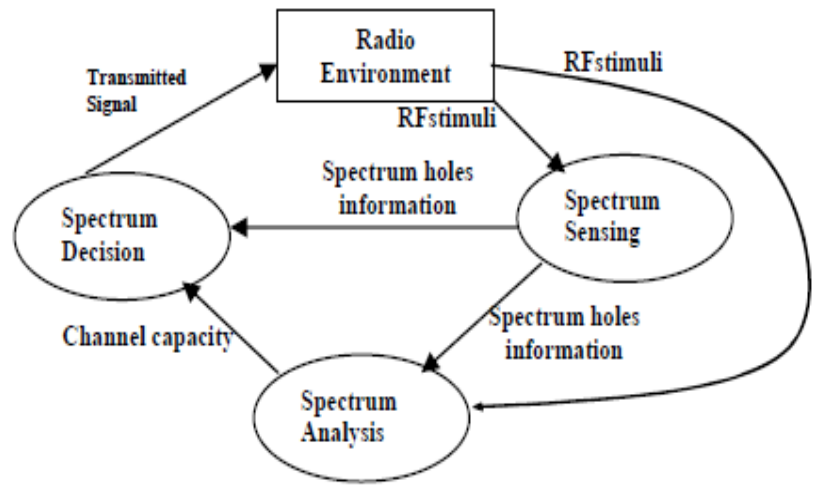

Fig.4: Cognitive Radio Process

The underutilized frequency bands of the radio spectrum, owned by legally licensed users (Primary Users), are referred as "Spectrum Holes" or "White Spaces". This can be formally defined as:

"A Spectrum Hole is a band assigned to a primary user, but at a time and unambiguous topographical location, the band is not used by the user."

In the Fig. 4 of Cognitive Radio Process a cognitive radio monitors spectrum bands, captures their information, and then detects the spectrum spaces. The characteristics of the spectrum spaces that are detected through spectrum sensing are estimated. Then, the appropriate spectrum band is chosen according to the spectrum characteristics and user requirements. Once the operating spectrum band is determined, the communication can be performed over this spectrum band. Cognitive radio scenario consists of two-user cognitive radio are shown in figure, we assume that each user knows only his channel and the unused spectrum through adequate sensing, The cognitive user will listen to the channel and, if sensed idle, will transmit during the voids.

The same approach is applied in [10] Fig.5; it has 'm' number of users. These users know their sub-bands of communication. 
According to available RF bands the Spectrum Sensing approach used to identify the channel state.

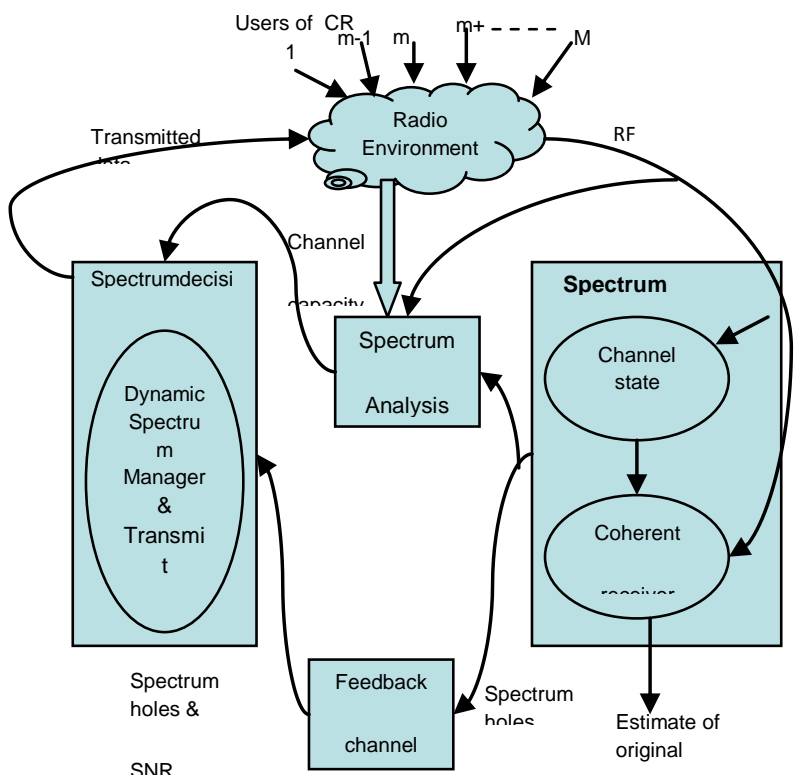

Fig.5: Cognitive Radio Process for $m$ users

The state of channel either lies into one of the given category as: Un-utilized, Partially utilized or fully utilized. Once the state has been identified the next role is to find out the user which is having one of the above states as well as to find out the requisite user for an application.

The state of sub-bands under un-utilized or partially utilized considered as Spectrum Holes or White Spaces. The information about the Spectrum Holes is conveyed to Spectrum Analysis directly and to Spectrum Decision via feedback channel. The Spectrum Decision also conveyed the information of SNR (Signal-to-Noise Ratio) at receiver input of user ' $m$ ' with the help of feedback channel. Thus, the feedback channel performs following roles adopted from Simon Hykins:

- Identifies the central frequencies and bandwidth of spectrum holes.

- Combined variance of interference plus thermal noise in each spectrum hole.

- Measure of output SNR (Signal-to-Noise Ratio) at the output of transmitter-receiver link, which is needed by the adaptive modulator in the transmitter.

There is also interfacing between the Radio Environment and the Spectrum Analysis. This interfaces the signal received from users $1,2,---, \mathrm{m}-1, \mathrm{~m}, \mathrm{~m}+1,---, \mathrm{M}$.

Spectrum Analysis provides the Channel Capacity to Spectrum decision block, which decides to which requisite user the sub-band has to govern. Once the information from Spectrum Analysis is obtained, Spectrum Decision block acts as Dynamic Spectrum Manager and Transmit Power Controller and Transmits the modulated message signal due to user 'm'.

\section{MODULATION TECHNIQUES}

A cognitive radio is a radio which can sense its environment and has the capability to adapt some of its features, such as carrier frequency, modulation, and transmission bandwidth and transmission power allowing dynamic reuse of the available spectrum. Due to fast setting out of various wireless systems, the restricted spectrum is becoming jam-packed increasingly. On the other hand, it is obvious that most of the allocated spectrum experience low utilization [9]. There is various modulation techniques used with Cognitive Radio like: Phase Shift Keying (PSK), Frequency shift Keying (FSK), Orthogonal Frequency Division Multiplexing (OFDM) and types of OFDM [12-16]. We are giving here a brief introduction about each modulation technique.

\subsection{Phase Shift Keying (PSK)}

Phase shift keying, PSK, is widely used these days within an entire propel of radio communications systems. It is entirely well suited to the growing area of data communications. PSK, Phase Shift Keying facilitates data to be carried on a radio communications signal in a more efficient manner than Frequency Shift Keying, FSK, and some other forms of modulation.

There are numerous tangs of Phase Shift Keying, PSK available. Each form has its own advantages and disadvantages, and a choice of the optimum system has to be made for each radio communications system that is designed. Only some of the major forms of PSK that are widely used in radio communications applications nowadays. Phase Shift Keying is a modulation technique in which the phase of the carrier wave is modified based on input signal to map data symbol to corresponding phase status. PSK is considered to be an efficient form of data modulation as it provides the lowest probability of error for a given received signal level, when it is measured over one symbol period. Various communication systems like Satellite communication systems and Terrestrial microwave radio links also operate on PSK as their modulation setup. If the phase of the signal is changed in accordance with the digital information data, then the modulation scheme is called Phase Shift Keying (PSK)[17]. The results observed in the form of BER and SNR using Phase Shift Keying Modulation technique concludes that data errors can be minimized using coding techniques, hence improving Signal to noise ratio. Hence, the signal can be recovered with very less probability of error at the destination [14].

\subsection{Convolutional Codes}

A major milestone in the history of convolution error correcting coding was the invention of maximum likelihood sequence estimation algorithm by Viterbi in 1967 [18]. Viterbi Algorithm did not resulted in minimum Bit Error rate; instead it finds the most likely sequence of transmitted bits. Convolution codes work on bit or symbol streams of arbitrary length Convolutional codes are frequently used to correct errors in noisy channels. They have rather good correcting capability and perform well even on very bad channels [19]. Viterbi Decoding is an optimal (in a maximum-likelihood sense) algorithm for decoding of a convolutional code. Its main drawback is that the decoding complexity grows exponentially with the code length. So, it can be utilized only for relatively short codes.

\subsection{Orthogonal Frequency Division Multiplexing (OFDM) and it's Variants}

The choice of physical layer transmission technique is a very important design decision when implementing a cognitive radio. To support high data-rate transmissions, the technique 
should be sufficiently agile to enable users to use a large bandwidth without interfering with incumbent users. Orthogonal frequency division multiplexing (OFDM) and multicarrier code division multiple access (MC-CDMA) are two high-speed modulation techniques employed in conventional transmission systems. OFDM has been shown to be an effective transmission technique for high speed data communications. On the other hand, MC-CDMA is capable of mitigating the effects of multiuser interference.

The popularity of the system lies in the fact that the modulation and demodulation can be performed simply by using the Inverse Fast Fourier Transform (IFFT) and Fast Fourier Transform (FFT). The time domain samples of an OFDM symbol can be calculated using an $\mathrm{N}$ point IFFT as equation (1):

$$
X_{n}=\sum_{K=0}^{N-1} X_{k} e^{j(2 \pi / N) n k}
$$

Where, $\mathrm{Xk}$ is the complex modulation value for the subcarrier with index $\mathrm{k}$. Then, prior to transmission, a Cyclic Prefix (CP) is added to each symbol. Although the channel equalization can be performed in a simple manner exploiting the $\mathrm{CP}$, this method reduces the effective data rate of the system [15]. There are some major drawbacks of OFDM are:

- To perform interference free demodulation the subcarriers have to remain orthogonal, otherwise system performance will degrade. Therefore, OFDM is very sensitive to frequency offset originating from the mismatch of transmitter and receiver local oscillators.

- The time domain OFDM signal is a sum of a large number of complex sinusoids, which means that, according to the central limit theorem, the amplitude distribution will be Gaussian, leading to a large PAPR of the signal. Hence, a power amplifier with a relatively large linear range is required; otherwise non-linear effects will severely degrade the system performance.

Other than OFDM, there are various versions of OFDM are used like Constant Envelope OFDM (CE-OFDM) is used to mitigate the PAPR (Peak-to-Average Power Ratio) of OFDM Signals [20], DFTS-OFDM systems [21], the complex modulation data set is preprocessed, the complex modulation values which will be transmitted are grouped and a Discrete Fourier Transform (DFT) is applied. Then the output of the DFT is used to modulate the subcarriers. This technique can be viewed as a single carrier modulation scheme, where frequency spreading is applied through all subcarriers. The comparison of OFDM, CE-OFDM and DFT-OFDM w.r.t. the PAPR is as:

Table 1: Comparison of Versions of OFDM.

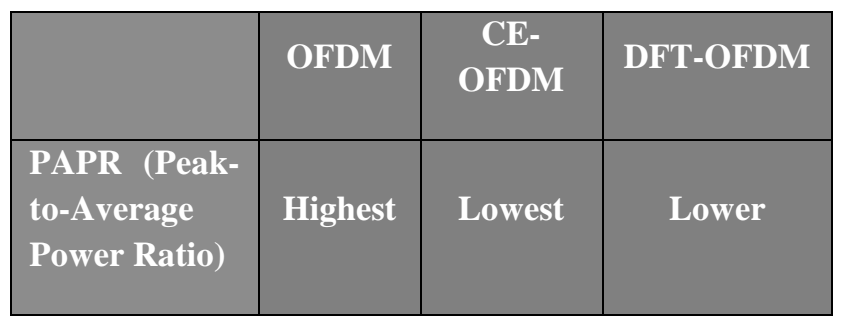

\section{SPECTRUM SENSING}

Detecting the unused spectrum and sharing it without harmful interference with other users. It is on important requirement of Cognitive Radio Networks to Sense Spectrum Holes or White Spaces. Detecting Primary Users (PUs) is the most efficient way to detect spectrum holes. Spectrum Sensing is corner stone of Cognitive Radio; it defines the availability of the spectrum for secondary Users (SUs) in Cognitive Radio Networks. The effectiveness of Spectrum Sensing determines the overall spectrum utilization.

The Primary users can be formally defined as :

"A user who has higher priority or legally rights on the usage of a specific part of the spectrum is the Primary User (PU)".

The Secondary User can also be defined as:

"The users which have lower priority, exploit this spectrum in such a way that they don't cause interference to Primary Users are the Secondary Users (SUs)".

Thus, the Secondary User need to have Cognitive Radio capabilities, to both "detect the White Spaces" and "to overlay" its own transmission on the white space making sure not to interfere with other primary users. Spectrum is therefore shared between the Primary users and Secondary users, with licensed Primary users have priority [22].

Spectrum Sensing detects the unused spectrum and shares it without harmful interference with other licensed users. A number of spectrum sensing methods have been proposed in literature. There are several factors that make spectrum sensing practically challenging.

- $\quad$ The required SNR for detection may be very low.

- Multi-path fading and time dispersion of wireless channels complicate the spectrum sensing problem.

- The noise level may change with time and location which succumbs the noise power uncertainty issue for detection.

There are several issues related to Spectrum sensing [23]-[25] like:

- Channel Uncertainty [24]: In wireless communication networks, qualms in received signal strength arises due to channel fading or shadowing which may wrongly construed that the primary system is located out of the secondary user's interference range as the primary signal may be experiencing a deep fade or being heavily shadowed by obstacles. Therefore, cognitive radios have to be more thoughtful to extricate a faded or shadowed primary signal from a spectrum hole. The higher sensitivity is required to avoid the uncertainty in received power of primary users.

- $\quad$ Noise Uncertainty [25]: The detection sensitivity can be denoted as the minimum SNR at which the primary signal can be accurately (e.g. with a probability of 0.99 ) detected by the cognitive radio and is given by [25],

$$
\mathrm{Ymin}=\{\mathrm{Pp} L(\mathrm{D}+\mathrm{R})\} / \mathrm{N}
$$

Where $\mathrm{N}$ is the noise power, $\mathrm{Pp}$ is transmitted power of the primary user, $\mathrm{D}$ is the interference range of the secondary user, and $\mathrm{R}$ is maximum distance between primary transmitter and its corresponding receiver. 


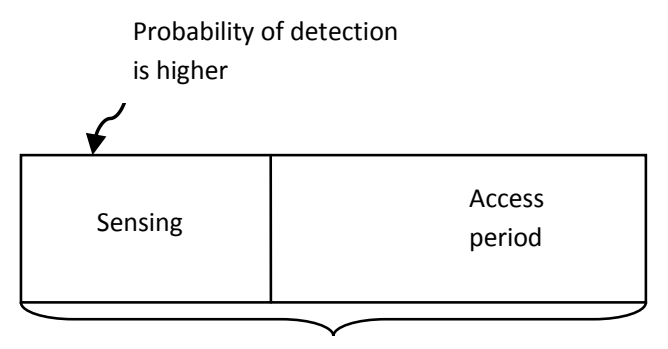

Spectrum access period of licensed user

\section{Long Observation period}

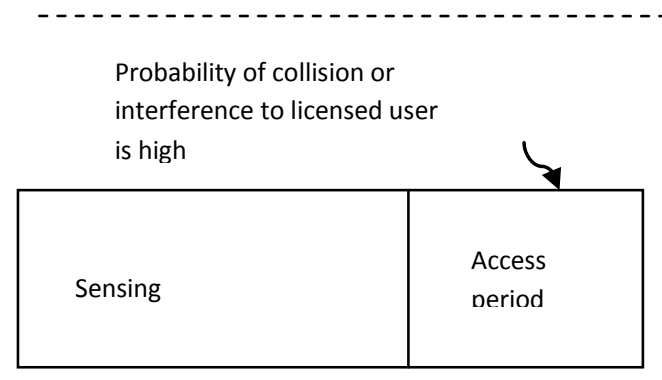

\section{Short Observation period}

Fig.6: Trades-off between sensing time and user throughput [24].

- Aggregate Interference Uncertainty: Due to the general deployment of secondary systems, there will be increased possibility of multiple cognitive radio networks functioning over the same licensed band. As a result, spectrum sensing will be affected by uncertainty in cumulative interference (e.g. due to the unknown number of secondary systems and their locations). When a primary system is out of interference range of a secondary system, the aggregate interference may lead to wrong detection. This uncertainty creates a need for more sensitive detector.

- Sensing Interference Limit: Primary goal of spectrum sensing is to detect the spectrum status i.e. whether it is idle or occupied, so that it can be accessed by an unlicensed user. The factors presents with the interference measurement at the licensed receiver caused by transmissions from unlicensed users.

- An unlicensed user may not know exactly the location of the licensed receiver which is required to compute interference caused due to its transmission.

- If a licensed receiver is a passive device, the transmitter may not be aware of the receiver. So these factors need attention while calculating the sensing interference limit.

Some issues are also related to Spectrum mobility [23],[26]:

- $\quad$ Search for the best frequency band: A cognitive radio must keep track of available frequency bands so that if necessary (e.g. a licensed user is detected), it can switch immediately to other frequency band.
- Protocol Stack Adaptation: the latency due to spectrum handoff could be high, the modification and adaptation of other components in the protocol stack is required.

- Self Coexistence \& Synchronization [26]: When an unlicensed (or secondary) user performs spectrum handoff, two issues have to be taken into account [26].

- $\quad$ The target channel must not currently be used by any other secondary user (i.e. the self coexistence requirement).

- The receiver of the corresponding secondary link must be notified of the spectrum handoff (i.e. the synchronization requirement).

For the self coexistence issue, a spectrum broker can be used to manage spectrum allocation. For synchronization, the MAC protocol must be designed with provision for spectrum handoff information exchange.

In this paper we are trying to accommodate the Spectrum Sensing methods are in following ways [24-31] Fig.7:

\subsection{Classical Spectrum Sensing or Primary Transmitter Detection}

Cognitive Radio must have the capability to determine if, a signal from a primary transmitter is locally present in a certain spectrum. There are several approaches available with Classical Spectrum Sensing. Some of them are explained here:

\subsubsection{Energy Detection [32]:}

One of the most commonly used approaches to detect the presence of transmission is based on energy detector, also known as Radiometer. It performs a measurement of the received energy in selected time and frequency range. Such measurement is compared with a threshold, which depend on the noise floor. The presence of a signal is detected when received energy is greater than an established threshold.

A Band pass filter can be applied to the received signal to measure the signal power in a particular frequency band. At ith Secondary User (SU), the statistic used for spectrum sensing is given by equation (2):

Where, rij is the jth sample of received signal at ith SU, and $\mathrm{N}=\mathrm{TW}$, where $\mathrm{T}$ and $\mathrm{W}$ respectively represents the detection time and the signal bandwidth in $\mathrm{Hz}$.

$$
E_{i}=\sum_{j=}^{N}\left|r_{i j}\right|^{2}
$$

Advantage: It has low computational complexity and without the need of the prior knowledge of the modulation types employed at Primary Users (PU).

Disadvantage: The lack of knowledge of the modulation type or data rate, the performance of Energy Detector could degrade significantly when the interference caused by another $\mathrm{PU}$ is presented in network.

In Energy Detector Based Sensing, the characterization of energy detection is done with the help of Hypothesis Test. 


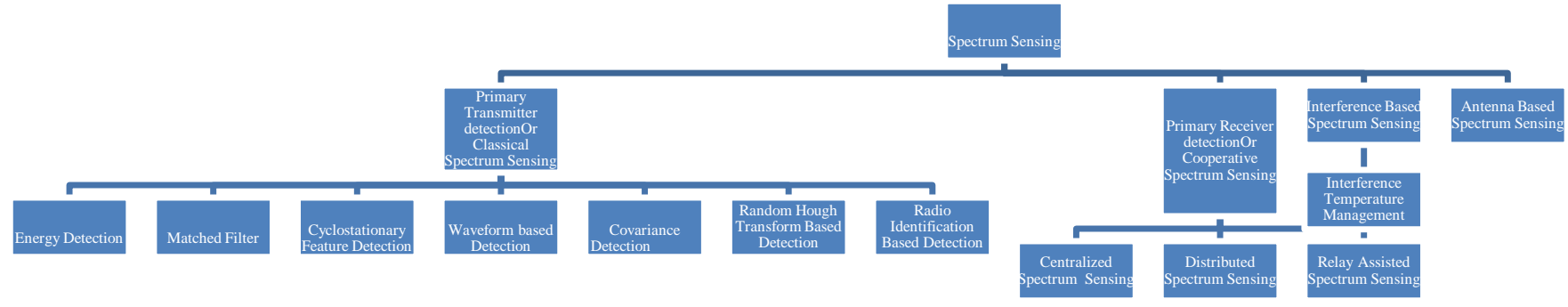

Fig. 7: Classification of Spectrum Sensing Methods [24-31]

\subsubsection{Pilot Detection via Matched Filter [33]:}

Cabric et al in [34] use matched filter for pilot detection. This method assumes that the primary user sends pilot signal with data. The pilot signal should be known by secondary users too allowing them to perform timing and carrier synchronization to achieve coherence [35]. Secondary users should have full prior knowledge of modulation type, pulse shaping and packet format. In this scenario, secondary users should provide separate dedicated receiver for each primary user class.

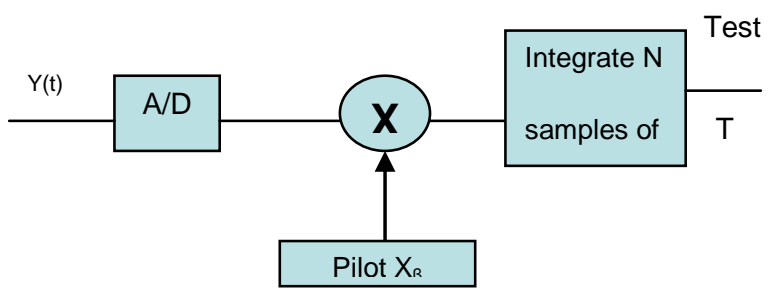

Fig.8: Pilot Detection via Matched Filter[33]

Advantage: Pilot detection requires minimum sensing time because it exploits available knowledge.

\section{Disadvantage:}

- Defenselessness to frequency offsets and the consequential loss of synchronization [35].

- The prior awareness of signal parameter to secondary users and providing a dedicated receiver corresponding to each primary user is completely impractical.

\subsubsection{Cyclostationary feature Detection:}

When a transmitted signal is modulated with a sinusoidal carrier, cyclic prefixes (as in OFDM), code or hopping sequences (as in CDMA); cyclostationarity is induced i.e. mean, autocorrelation show periodic behavior. This feature is exploited in a Cyclostationary Feature Detector that measures a signal property called Spectral Correlation Function.

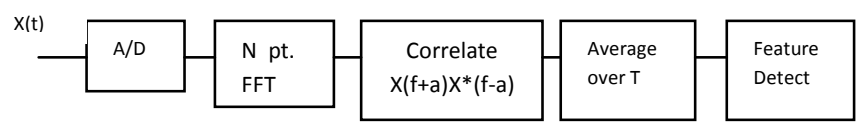

Fig.9: Cyclostationary Spectrum Sensing

Advantage: Cyclostationary Spectrum Sensing performs better than Energy detection because of its noise rejection ability. This occurs because noise is totally random and does not exhibit any periodic behavior. When we have no prior knowledge about primary user's waveform which is the scenario in real life, then best technique is Cyclostationary feature detection.

Disadvantage: It has high complexity which results in high cost.

\subsubsection{Covariance Detection:}

The main aim of Covariance based Spectrum Sensing is to exploits the Covariance of signal as wall as noise. Statistically both the things are different. This property is used to differentiate signal from noise where the sample covariance matrix of the received signal is computed based on the receiving filter.

$$
\mathbf{Y}=\mathbf{H s}+\mathbf{w}
$$

Where, $\mathrm{Y}$ is the Received Signal matrix, $\mathrm{H}$ is the channel matrix, S is the Signal matrix and W is the Noise matrix.

The covariances corresponding to the signal and noise can be written as:

$$
\mathbf{R y}=\mathbf{E}\left[\mathbf{y y}_{\dagger}^{\dagger}\right] ; \mathbf{R s}=\mathbf{E}[\mathbf{s s} \dagger] ; \mathbf{R n}=\mathbf{E}[\mathbf{w w} \dagger]
$$


If there is no signal, then Rs $=0$. Hence the off-diagonal elements of Ry are all zeros. If there is signal and the signal samples are correlated, Rs is not a diagonal matrix. Hence, some of the off-diagonal elements of Ry should not be zeros. Therefore, this method detects the presence of signals with the help of covariance matrix of the received signal.

\subsubsection{Waveform Based Spectrum Sensing:}

The patterns corresponding to the signal, such as preambles, midambles, regularly transmitted pilot patterns, spreading sequences, etc, are usually utilized in wireless systems to assist synchronization or detect the presence of signal. When a known pattern is present, the detection method by correlating the received signal with a known copy of itself [36] can be performed and the method is known as waveform-based detection.

Advantage: The performance of Waveform Based Detection is better than the Energy Based Detection in terms of Reliability and Convergence Time.

\subsubsection{Random Hough Transform Based Detection:}

The Hough transform is widely used for pattern (such as lines, circles) detection in image processing. Recently, Random Hough transform has been proposed for CR in [37], where the Random Hough transform of received signal $y(n)$ is used for identifying the presence of radar pulses in the operating channels of IEEE 802.11 wireless systems.

\subsubsection{Radio Identification Based detection:}

In radio identification approach, several features such as transmission frequency, transmission range, modulation technique, etc, are extracted from the received signal $y(n)$ as,

$$
\mathrm{y}(\mathrm{n})=\mathrm{s}(\mathrm{n})+\mathrm{w}(\mathrm{n}) \ldots \ldots \ldots .(6)
$$

Where, $y(n)$ is the received signal, $s(n)$ is the transmitted signal, $\mathrm{w}(\mathrm{n})$ noise added to the signal, Which are used for selecting the most suitable secondary user technology for the $\mathrm{CR}$ transmission.

\subsection{Cooperative Spectrum Sensing or Primary Receiver Detection}

The process of cooperative sensing starts with spectrum sensing performed individually at each CR user called local sensing.

In Fig.10, [29] CR1 and CR2 are located inside the transmission range of primary transmitter (PU TX) while CR3 is outside the range. Due to multiple attenuated copies of the PU signal and the blocking of a house, CR2 experiences multipath and shadow fading such that the PU's signal may not be correctly detected. Moreover, CR3 suffers from the receiver uncertainty problem because it is unaware of the PU's transmission and the existence of primary receiver (PU $\mathrm{RX}$ ). As a result, the transmission from CR3 may interfere with the reception at PU RX. However, due to spatial diversity, it is unlikely for all spatially distributed CR users in a CR network to concurrently experience the fading or receiver uncertainty problem.

If CR users, most of which observe a strong PU signal like $\mathrm{CR} 1$ in the figure, can cooperate and share the sensing results with other users, the combined cooperative decision derived from the spatially collected observations can overcome the deficiency of individual observations at each CR user. Thus, the overall detection performance can be greatly improved. This is why cooperative spectrum sensing (simply called cooperative sensing thereafter) is an attractive and effective approach to combat multipath fading and shadowing and mitigate the receiver uncertainty problem.

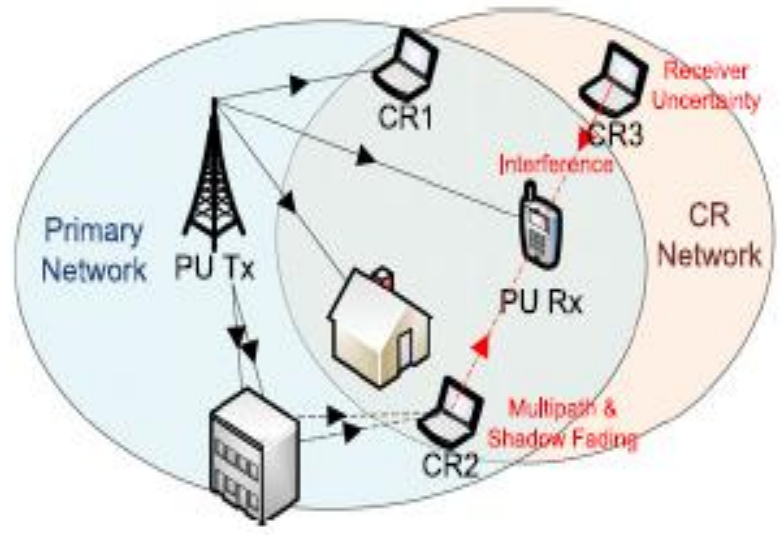

Fig.10: Receiver Uncertainty and multipath/shadow fading [29]

The Cooperative Sensing is classified into Centralized sensing [38-40], Distributed Sensing [41] and Relay Assisted Sensing [42-44]

\subsubsection{Centralized Cooperative Sensing:}

In centralized cooperative sensing, a central identity called Fusion Center (FC) (also known as base station, common receiver, combining node, master node, designated controller etc.) controls the three-step process of cooperative sensing.

- The FC selects a channel or a frequency band of interest for sensing and instructs all cooperating CR users to individually perform local sensing.

- All cooperating CR users report their sensing results via the control channel.

- Then, the FC combines the received local sensing information, determines the presence of PUs, and diffuses the decision back to cooperating CR users.

As shown in Fig.11, CR0 is the FC and CR1-CR5 are cooperating $\mathrm{CR}$ users performing local sensing and reporting the results back to CR0. For local sensing, all CR users are tuned to the selected licensed channel or frequency band where a physical point-to-point link between the PU transmitter and each cooperating CR user for observing the primary signal is called a sensing channel.

For data reporting, all CR users are tuned to a control channel where a physical point-to-point link between each cooperating $\mathrm{CR}$ user and the FC for sending the sensing results is called a reporting channel. The centralized cooperative sensing can occur in either centralized or distributed CR networks. In centralized CR networks, a CR base station (BS) is naturally the FC. Alternatively, in CR ad hoc networks (CRAHNs) where a CR BS is not present, any CR user can act as a FC to coordinate cooperative sensing and combine the sensing information from the cooperating neighbors. 


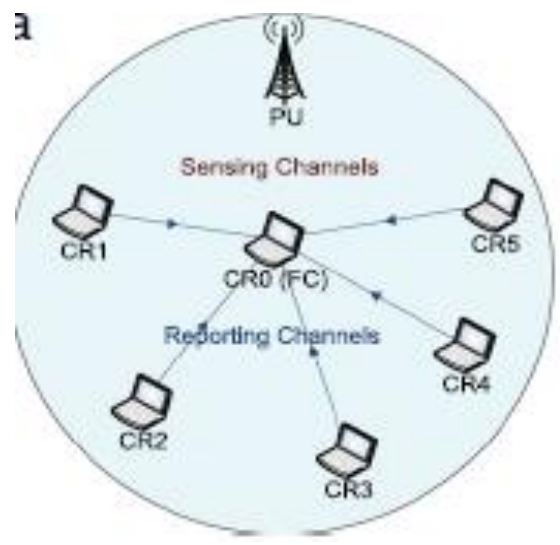

Fig. 11: Centralized Cooperative Sensing

\subsubsection{Distributed Cooperative Sensing}

Distributed cooperative sensing does not rely on a FC for making the cooperative decision.

Tis type of sensing follows the unite way between them. Cognitive Radio users communicate with each other between them in a united way. The united signal is unite to unified decision on the presence or absence of Pus by iterations. Fig.12, exemplifies the cooperation in the distributed manner. After local sensing, CR1-CR5 shares the local sensing results with other users within their transmission range. On the basis of a distributed algorithm, each CR user sends its own sensing data to other users, combines its data with the received sensing data, and decides whether or not the PU is present by using a local criterion. If the criterion is not satisfied, CR users send their combined results to other users again and repeat this process until the algorithm is converged and a decision is reached. In this manner, this distributed scheme may take several iterations to reach the unanimous cooperative decision

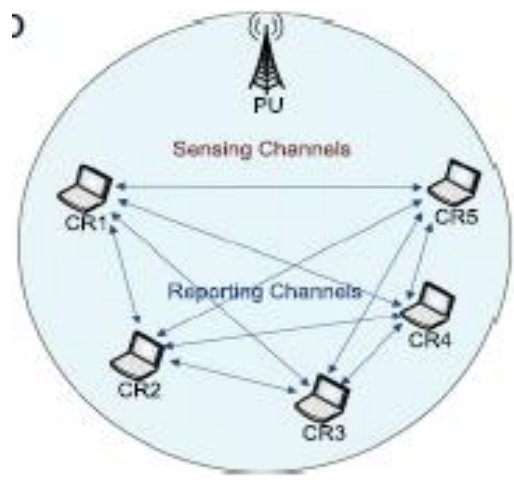

Fig.12: Distributed Cooperative Sensing.

\subsubsection{Relay Assisted Cooperative Sensing}

In addition to Centralized and Distributed Cooperative Sensing, the third scheme is relay-assisted cooperative sensing. Since both sensing channel and report channel are not perfect, a CR user observing a weak sensing channel and a strong report channel and a CR user with a strong sensing channel and a weak report channel, for example, can complement and cooperate with each other to improve the performance of cooperative sensing.

In Fig.13, CR1, CR4, and CR5, who observe strong PU signals, may suffer from a weak report channel. CR2 and CR3, who have a strong report channel, can serve as relays to assist in forwarding the sensing results from CR1, CR4, and CR5 to the FC.

In this case, the report channels from CR2 and CR3 to the FC can also be called relay channels. Note that although Fig. 13 shows a centralized structure, the relay-assisted cooperative sensing can exist in distributed scheme.

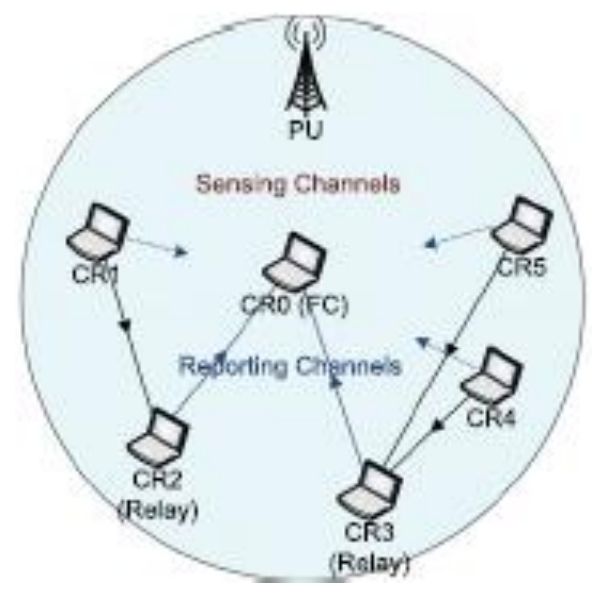

Fig.13: Relay Assisted Cooperative Sensing

In fact, when the sensing results need to be forwarded by multiple hops to reach the intended receive node, all the intermediate hops are relays. Thus, if both centralized and distributed structures are one-hop cooperative sensing, the relay-assisted structure can be considered as multi-hop cooperative sensing. In addition, the relay for cooperative sensing here serves a different purpose from the relays in cooperative communications [45], where the CR relays are used for forwarding the PU traffic.

\section{Advantages of Cooperative Spectrum Sensing:}

- The plummeting sensitivity requirements: Channel impairments like multipath fading, shadowing and building penetration losses, impose high sensitivity requirements inherently limited by cost and power requirements. Employing cooperation between nodes can drastically reduce the sensitivity requirements up to -25 $\mathrm{dBm}$, also reduction in sensitivity threshold can be obtained by using this scheme;

- Agility improvement: All topologies of cooperative networks reduce detection time compared to uncoordinated networks.

- $\quad$ Reduced false alarms

- More accurate signal detection.

Disadvantage:

- The CR users need to perform sensing at periodic intervals as sensed information become obsolete fast due to factors like mobility, channel impairments etc. This considerably increases the data overhead; large sensory data: since the cognitive radio can potentially use any spectrum hole, it will have to scan a wide range of spectrum, resulting in large amounts of data, being inefficient in terms of data throughput, delay sensitivity requirements and energy consumption. Even though cooperatively sensing data poses lot of challenges, it could be carried out without incurring much overhead because only approximate sensing information is required, eliminating the need for complex signal 
processing schemes at the receiver and reducing the data load.

- Even though a wide channel has to be scanned, only a portion of it changes at a time requiring updating only the changed information and not all the details of the entire scanned spectrum.

- $\quad$ Significant requirements are:

$$
\begin{array}{ll}
\circ & \text { Control Channel } \\
\circ & \text { System Synchronization } \\
\circ & \begin{array}{l}
\text { Suitable geographical spread of cooperating } \\
\text { nodes. }
\end{array}
\end{array}
$$

\subsection{Interference Based Sensing}

In the Interference Based Sensing CR users would operate in spectrum underlay (UWB like) approach. The Interference Temperature Management scheme comes under the Interference Based Sensing.

\subsubsection{Interference Temperature Management}

The basic idea behind the interference temperature management is to set up an upper interference limit for given frequency band in specific geographic location such that the CR users are not allowed to cause harmful interference while using the specific band in specific area. Typically, CR user transmitters control their interference by regulating their transmission power (their out of band emissions) based on their locations with respect to primary users. This method basically concentrates on measuring interference at the receiver.

The operating principle of this method is like an UWB technology where the CR users are allowed to coexist and transmit simultaneously with primary users using low transmit power that is restricted by the interference temperature level so as not to cause harmful interference to primary users.

Limitations: CR users do not perform spectrum sensing for spectrum opportunities and can transmit right way with specified preset power mask. However, the CR users can not transmit their data with higher power even if the licensed system is completely idle since they are not allowed to transmit with higher than the preset power to limit the interference at primary users. It is noted that the CR users in this method are required to know the location and corresponding upper level of allowed transmit power levels. Otherwise they will interfere with the primary user transmissions.

\subsection{Antenna Based Spectrum Sensing}

The spectrum can be sense by single antenna (SISO - Single Input Single Output) or multiple antennas (SIMO - Single Input Multiple Output, MIMO- Multiple Input Multiple Output). The challenging and emerging area in antenna based spectrum sensing is sensing through multiple antennas.

\subsubsection{Multiple Antenna Spectrum Sensing (SIMO)} It is a new area in cognitive radio; it explains that if multiple antennas are mounted at secondary user then high prospects of detection is obtained. In [47] author proposes a Generalized Likelihood Ratio detector (GLRD) which do not have any primal information about the parameters like; channel gains, noise variance, and primary user signal variance. It is supposed that the SU has $\mathrm{M}$ receiving antennas and each antenna receives $\mathrm{L}$ samples. It is also assumed that the PU signal samples are independent zero-mean random variables with complex Gaussian distribution.

The benefits of MIMO are as:

- Higher capacity (bits/s/Hz). (spectrum is expensive; number of base stations limited)

- Better transmission quality

- Increased coverage

- Improved user position estimation

These benefits are achieved due to spatial multiplexing gain, diversity gain, and array gain and interference detection.

In case of multiple antenna spectrums sensing there is one antenna at primary user and multiple antennas at secondary user; this is the case of SIMO (single input and multiple output).

\subsubsection{MIMO based spectrum sensing}

In MIMO spectrum sensing, multiple antennas are employed at both on primary user and secondary user.

This type of spectrum sensing is done in two ways:

- For Independent channel

- For Correlated channel

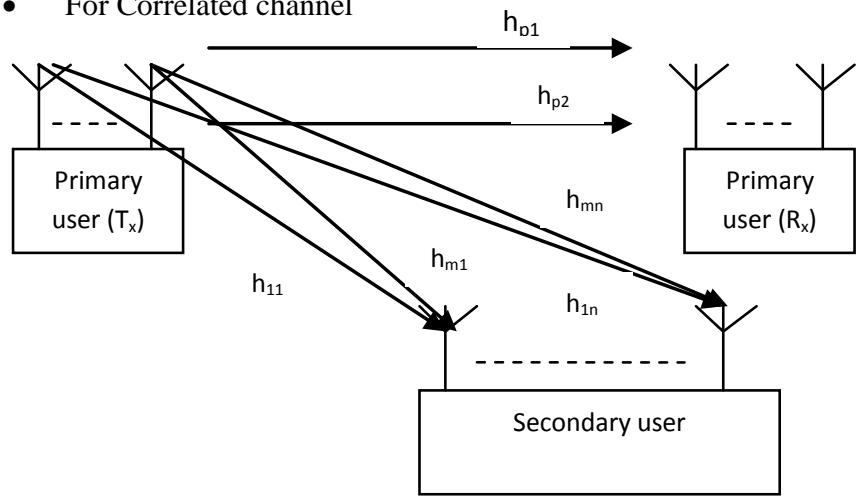

Fig. 14: MIMO based Spectrum Sensing.

\subsection{Other Sensing Techniques}

Some other methods of Spectrum sensing are kept in to this category:

\subsubsection{Wavelet Based Detection [48-49]}

By precisely locating the spectrum boundaries one can effectively prevent the problem of interference between adjacent bands and increase the data rates in the channel. Of various attempts that have been made to accurately detect the spectral boundaries, Wavelet Edge detection is a method which is widely utilized; however the efficiency of this system is highly dependent upon the type of wavelet used. The Wavelet Based Spectrum Sensing is done in many ways:

\subsubsection{Multiscale Product}

This method involves finding discontinuities in PSD of the signal by taking the multi-scale wavelet transforms and estimating the edges as explained in Section 3.3. This is done under the assumption that the discontinuities in the Power Spectral Density represent the spectral boundaries. The energy 
of each sub-band is calculated for spectrum occupancy. This method also assumes that there is always an empty sub-band and hence noise band can be determined.

\subsubsection{Discrete Wavelet Packet Transform (DWPT)}

DWPT employs two sets of functions, called scaling functions and wavelet functions, which are associated with low pass and high pass filters, respectively. The decomposition of the signal into different frequency bands is simply obtained by successive high pass and low- pass filtering of the time domain signal. In [48] the original signal $x[n]$ is first passed through a half band high pass filter $\mathrm{g}[\mathrm{n}]$ and a low pass filter $\mathrm{h}[\mathrm{n}]$. After the filtering, half of the samples can be eliminated according to the Nyquist's rule, since the signal now has a highest frequency of $J / 2$ radians instead of $J$. The signal can therefore be subsampled by 2 , simply by discarding every other sample. This constitutes one level of decomposition and can mathematically be expressed as follows:

$$
\begin{gathered}
y_{\text {high }}[\mathrm{k}]=\sum_{\mathrm{n}} \mathrm{x}[\mathrm{n}] * \mathrm{~g}[2 \mathrm{k}-\mathrm{n}] \\
y_{\text {low }}[\mathrm{k}]=\sum \mathrm{x}[\mathrm{n}] * \mathrm{~h}[2 \mathrm{k}-\mathrm{n}]
\end{gathered}
$$

Where, yhigh $[\mathrm{k}]$ and ylow $[\mathrm{k}]$ are the outputs of the high pass and low pass filters respectively, after sub sampling by 2 .

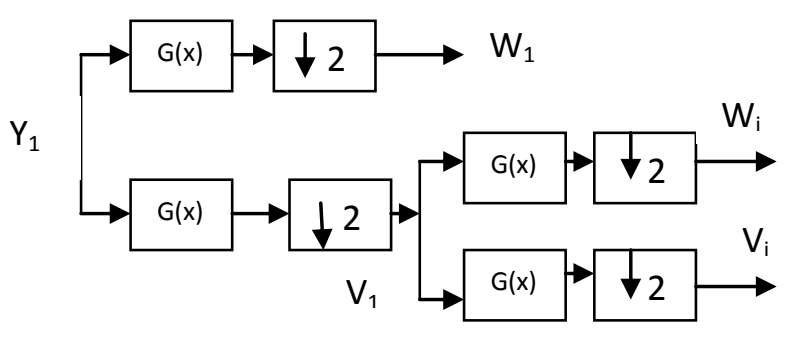

Fig.15: Decomposition of signal in different frequency bands $[48]$

The Discrete Wavelet Packet Transform is a better edge detection method than Continuous Wavelet Transform (CWT) based method. The wavelet packet transform is just like the wavelet transform except that it decomposes even the high frequency bands which are kept intact in the wavelet transform.

\subsubsection{Multi Taper Spectrum Sensing or Estimation (MTSE)}

The Multi Taper spectrum estimation (MTSE) is proposed in [50], where the last $\mathrm{N}$ received samples are collected in a vector form and represented it as a set of slepian base vectors. The fundamental property of Slepian vectors is that their Fourier transforms have the maximal energy concentration in the bandwidth $\mathrm{fc}-\mathrm{W}$ to $\mathrm{fc}+\mathrm{W}$ under a finite sample-size constraint. Using this feature, CR user can identify the spectrum opportunity.

\subsubsection{Filter Bank Based Spectrum Sensing or Estimation (FBSE)}

The filter bank based spectrum estimation (FBSE) has been proposed for multicarrier modulation based CR systems by using a pair of matched-root Nyquist-filter. In [51], FBSE has been compared with MTSE, and noted that FBSE outperforms MTSE with low PSD since MTSE uses multiple prototype filters for a given frequency band and the best response corresponds to the largest eigen value of the auto-correlation matrix of the observation vector. Moreover, MTSE is better for small sample spaces whereas FBSE is better for large number of samples [51]. However the MTSE approach increases the computational complexity and hence might not be suitable for CR system.

Table.2: Comparison between FBSE and MTSE

\begin{tabular}{|l|l|l|}
\hline Parameters & FBSE & MTSE \\
\hline $\begin{array}{l}\text { Power Spectral Density } \\
\text { (PSD) }\end{array}$ & LOW & HIGH \\
\hline $\begin{array}{l}\text { Performance with large } \\
\text { Sample Space }\end{array}$ & GOOD & NOT GOOD \\
\hline \multicolumn{1}{|c|}{ Complexity } & LOW & HIGH \\
\hline
\end{tabular}

\section{CONCLUSION}

The limited spectrum for dense wireless communications and inefficient spectrum utilization necessitate a new communication paradigm cognitive radio which can exploit the unutilized spectrum opportunistically. This paper presents some of the cognitive radios issues used to determine the effectiveness in wireless communication. These characteristics are crucial when applying the cognitive radios in order to determine the effectiveness and reliability of wireless networks. Here, we discussed some Physical layer aspects of cognitive Radio like: Types of Cognitive Radio, Architectures of Cognitive Radio, Cognitive Process, Different Modulation Techniques used for signal transmission in $\mathrm{CR}$ and various Spectrum Sensing techniques. Many researchers are using their efforts in finding the best solutions for the routing protocols, cross-layer design approaches and Cognitive Radio security issues. The recent and evolving research efforts have made big progress on cognitive radios both in theory and in practical implementations.

\section{REFERENCES}

[1] Yang Xiao, Fei Hu "Cognitive Radio Networks" edited by CRC Press.

[2] Ashwin Amanna, Jeffrey H. Reed, "Survey of Cognitive Radio Architecture"

[3] J. Mitolla, "Cognitive Radio - An Integrated Agent Architecture for Software Defined Radio," Royal Institute of Technology (KTH), 2000.

[4] B. Le, F. A. G. Rodriguez, Q. Chen, B. P. Li, F. Ge, M. ElNainay, T. W. Rondeau, and C. W. Bostian, "A Public Safety Cognitive Radio Node," in 2007 SDR Forum Technical Conference Denver, CO, 2007.

[5] P. Kyasanur and N. Vaidya, "Routing in multichannel multi-interface ad hoc wireless networks," Tech. rep., UIUC, Dec. 2004.

[6] A. Raniwala, K. Gopalan, and T. cker Chiueh, "Centralized channel assignment and routing algorithms for multi-channel wireless mesh networks," SIGMOBILE Mob. Comp. Comm. Rev., 8(10.2):50-65, 2004. 
[7] G. Cheng, W. Liu, Y. Li, and W. Cheng, "Spectrum aware on-demand routing in cognitive radio networks," Proc. of IEEE DySPAN 2007.

[8] Amna Saad Kamil,I brahim Khider, "Open Research issues in Cognitive Radio" 16 Telecommunication Forum TELFOR, Serbia, Belgrade pp. 250-253, November 25-27, 2008.

[9] Federal Communications Commission, "Facilitating opportunities for flexible, efficient, and reliable spectrum use employing cognitive radio technologies, notice of proposed rulemaking and order, FCC 03-322," Dec. 2003.

[10] S. Haykin, "Cognitive radio: Brain empowered Wireless communications," IEEE J. Select. Areas Commun., vol. 23, no. 2, pp. 201-220, Feb. 2005.

[11] Vaclav Valenta, Roman Marsalek," Survey on Spectrum Utilization in Europe: Measurements, Analyses and Observations," 5th International ICST Conference on Cognitive Radio Oriented Wireless Networks and Communications, Cannes, France, 2010.

[12] Zsolt Kollar, Peter Horvath, "Modulation Schemes for Cognitive Radio in White Spaces", RADIOENGINEERING, vol. 19, no. 4, pp. 511-517, December, 2010.

[13] Ibrahim Budiarjo, Homayoun Nikookar, and Leo P. Ligthart, "Conitive Radio Modulation Techniques", IEEE Signal Processing Magazine, pp. 24-34, November 2008.

[14] Ritu Khullar, Sippy Kapoor, Naval Dhawan, "Modulation Technique for Cognitive Radio Applications", International Journal of Engineering Research and Applications (IJERA), Vol. 2, Issue 3, pp.123-125, May-Jun 2012.

[15] Zsolt Kollar, Peter Horvath, "Physical Layer Considerations For Cognitive Radio: Modulation Techniques".

[16] Rakesh Rajbanshi Qi Chen Alexander M. Wyglinski Gary J. Minden Joseph B. Evans, "Quantitative Comparison of Agile Modulation Techniques for CognitiveRadio Transceivers".

[17] Daniel Warne, "Software Radio Architectures-Part 2", 2004.

[18] Chip Fleming, "A Tutorial on Convolutional Coding with Viterbi Decoding”, 2002.

[19] Davoud Arasteh, "Teaching Convolutional Coding using MATLAB" in Communication Systems Course, Proceedings of the ASEE Gulf South-west Annual Conference Southern University, Baton Rouge,2006.

[20] S. C. Thompson and A. U. Ahmed "Constant-envelope OFDM", IEEE Trans. On Commun., Vol. 56, No. 8, Aug. 2008.

[21] Hyung G. Myung, Junsung Lim, and David J. Goodman "Single Carrier FDMA for Uplink Wireless Transmission", IEEE Vehicular Technology Magazine, Vol. 1, No. 3, pp. 30-38, Sep. 2006.

[22] Linda Doyle, "Essential of Cognitive Radio", Cambridge University Press.
[23] Mansi Subhedar, Gajanan Birajdar, "Spectrum Sensing Techniques in Cognitive Radio: A Survey", International Journal of Next Generation Networks (IJNGN), vol.3, no.2, pp. 37-51 June, 2011.

[24] Ekram Hossain, Dusit Niyato, Zhu Han, "Dynamic Spectrum Access and Management in Cognitive Radio Networks", Cambridge University Press, 2009.

[25] D. B. Rawat, G. Yan, C. Bajracharya, "Signal Processing Techniques for Spectrum Sensing in Cognitive Radio Networks", International Journal of Ultra Wideband Communications and Systems, Vol. x, No. x/x, pp:1-10, 2010.

[26] Brodersen, R., Wolisz, A., Cabric, D., Mishra, S., Willkomm, D., "CORVUS: A Cognitive radio Approach for Usage of Virtual Unlicensed Spectrum", Berkeley Wireless Research Center, white paper 2004

[27] Hano Wang, Gosan Noh, Dongkyu Kim, Sungtae Kim, and Daesik Hong, "Advanced Sensing Techniques of Energy Detection in Cognitive Radios", Journal of Communications and Networks, vol. 12, no. 1, pp. 19-29 February 2010.

[28] Nisha Yadav and Suman Rathi, "A Comprehensive Study Of Spectrum Sensing Techniques In Cognitive Radio", International Journal of Advances in Engineering \& Technology, IJAET, Vol. 1, Issue 3, pp.85-97 July 2011.

[29] Ian F. Akyildiz, Brandon F. Lo , Ravikumar Balakrishnan, "Cooperative spectrum sensing in cognitive radio networks: A survey", Elsevier Physical Communication vol. 4 , pp. 40-62, 2011.

[30] Danda B. Rawat and Gongjun Yan, "Signal Processing Techniques for Spectrum Sensing in Cognitive Radio Systems: Challenges and Perspectives", IEEE, 2009.

[31] Hemalatha.M, Prithviraj.V, Jayalalitha.S, Thenmozhi.K, Bharadwaj.D, Girish G.Koundinya, "A Survey Report On Spectrum Sensing Techniques In Cognitive Radio", Journal of Theoretical and Applied Information Technology, Vol. 37 No.1, pp. 32-38, March 2012.

[32] Jian-Lang Gao, “ A Distributed Detection Scheme by Combining Energy Detectors and Cyclostationary-based Detectors for Power Spectrum Sensing Cognitive Radio", June 2009.

[33] D.D.Ariananda, M.K.Lakshmanan, H.Nikookar, "A Survey on Spectrum Sensing Techniques for Cognitive Radio", CogART'09, pp 74-79, 2009.

[34] D. Cabric, S. M. Mishra, and R. W. Brodersen, "Implementation Issues in Spectrum Sensing for Cognitive Radios", Proceedings of the Asilomar Conference on Signals and Systems, 2004.

[35] D. Cabric, A. Tkachenko, R.W. Brodersen, "Spectrum Sensing Measurements of Pilot, Energy, and Collaborative Detection", IEEE Military Communications Conference, October, 2006. Tang, "Some Physical Layer Issues of Wide-band Cognitive Radio Systems," in IEEE Int. Symposium on New Frontiers in Dynamic Spectrum Access Networks, Baltimore, MD, pp. 151-159, Jun. 2005.

[36] K. Challapali, S. Mangold, and Z. Zhong, "Spectrum Agile Radio: Detecting Spectrum Opportunities," in 
Proc. Int. Symposium on Advanced Radio Technologie, Boulder, CO, Mar. 2004.

[37] A. Ghasemi, E. Sousa, "Collaborative spectrum sensing for opportunistic access in fading environments", in: Proc. of IEEE DySPAN 2005, pp. 131-136, 2005.

[38] E. Visotsky, S. Kuffner, R. Peterson, "On collaborative detection of TV transmissions in support of dynamic spectrum sharing", in: Proc. Of IEEE DySPAN 2005, pp. 338-345, 2005.

[39] J. Unnikrishnan, V.V. Veeravalli, "Cooperative sensing for primary detection in cognitive radio", IEEE Journal of Selected Topics in Signal Processing 2 (1) pp.18-27, 2008.

[40] Z. Li, F. Yu, M. Huang, "A cooperative spectrum sensing consensus scheme in cognitive radios", in: Proc. of IEEE Infocom 2009, pp. 2546-2550, 2009.

[41] G. Ganesan, Y.G. Li, "Cooperative spectrum sensing in cognitive radio-part I: two user networks", IEEE Transactions on Wireless Communications 6 (6), 22042213, 2007.

[42] G. Ganesan, Y.G. Li, "Cooperative spectrum sensing in cognitive radio-part II: multiuser networks", IEEE Transactions on Wireless Communications 6 (6), 22042213, 2007.

[43] G. Ganesan, Y.G. Li, "Cooperative spectrum sensing in cognitive radio-part II: multiuser networks", IEEE Transactions on Wireless Communications 6 (6), 22042213, 2007.

[44] W. Zhang, K. Letaief, "Cooperative spectrum sensing with transmit and relay diversity in cognitive radio network"s - [transaction letters], IEEE Transactions on Wireless Communications 7 (12), 4761-4766, 2008.
[45] K. Ben Letaief, W. Zhang, "Cooperative communications for cognitive radio networks", Proceedings of the IEEE 97 (5) 878-893, 2009.

[46] F. Zeng, Z. Tian, C. Li, "Distributed compressive wideband spectrum sensing in cooperative multi- hop cognitive networks", in: Proc. of IEEE ICC 2010, pp: 1$5,2010$.

[47] Abbas Taherpour, Masoumeh Nasiri-Kenari, and Saeed Gazor,"Multiple antenna spectrum sensing in cognitive radios",IEEE Transactions on Wireless Communications, vol. 9 , (February 2010), pp. 814- 823, 2010.

[48] Varadharajan. E, Rajkumari. M, "Discrete Wavelet Transform Based Spectrum Sensing In Cognitive Radios Using Eigen Filter", International Journal of Advanced Engineering Technology, IJAET, Vol.III, Issue I, 389391, January-March, 2012.

[49] Karthik Divakaran, Naveen Manikandan P, Shri Hari R R, "Wavelet Based Spectrum Sensing Techniques For Cognitive Radio - A Survey", International Journal of Computer Science \& Information Technology (IJCSIT), Vol 3, No 2, pp. 123-137, April 2011.

[50] D. J. Thomson, "Spectrum Estimation and Harmonic Analysis," Proc. IEEE, vol. 20, pp. 1055-1096, Sep. 1982.

[51] B.Farhang-Boroujeny and R. Kempter, "Multicarrier Communication Techniques for Spectrum Sensing and Communications in Cognitive Radios," IEEE Communication Magazine, vol. 48, no. 4, Apr. 2008. 